\title{
Breast Augmentation for Ptosis: Effective Upward Rotation of the Nipple
}

\section{Jinseok Park}

Parkjinseok Aesthetic Clinic, Seoul, Korea

No potential conflict of interest relevant to this article was reported.
Background For the correction of small ptotic breasts, augmentation mastopexy provides a better shape than other techniques, but its periareolar or vertical scar is a limiting factor. Subglandular augmentation tends to increase ptosis. Submuscular augmentation may result in a "Waterfall" deformity (double contour deformity). This report uses examples of two patients to illustrate concepts for improving breast augmentation of ptotic breasts.

Methods Two patients initially underwent insertion of breast implants for ptotic breasts, using full height type of implants inserted by the type III dual plane technique. Both patients had wanted breasts that were not excessively large, but which had an improved shape and minimal scars. At nearly the same time after the initial surgery (postoperative day 3 and 7), each patient exhibited a unilateral double contour breast deformity. The initial implants were changed to shorter height implants to shift volume to the lower pole.

Results Changing the implants resulted in improved breast shape by enhancing the leverage effect. The double contour deformities observed after using the taller implants were improved by changing to shorter implants.

Conclusions For rotating the nipple areolar complex upward during correction of small ptotic breasts with implants, the type III dual plane technique is an effective way to produce a larger volume pocket in the lower pole of the breast. More leverage effect can be obtained by using a shorter height and greater projection type of shaped implant instead of a full height implant.

Keywords Breast, Breast implantation, Mammaplasty, Postoperative complications

\section{INTRODUCTION}

For the correction of small ptotic breasts, augmentation mastopexy produces a better shape than other techniques, but its periareolar or vertical scar is a limiting factor. Subglandular augmentation with an implant can improve the shape of the breast, but it tends to increase the ptosis, and in patients with scant breast tis-

Received: Jun 1, 2014 Revised: Jun 11, 2014 Accepted: Jun 12, 2014

Correspondence: Jinseok Park Parkjinseok Aesthetic Clinic,

B 111, JB Miso Bd. 841 Nonhyeon-ro, Gangnam-gu, Seoul 135-892, Korea

E-mail: breastpark@naver.com

Copyright @ 2014 The Korean Society for Aesthetic Plastic Surgery.

This is an Open Access article distributed under the terms of the Creative Commons Attribution Non-Commercial License (http://creativecommons.org/licenses/by-nc/3.0/) which permits unrestricted non-commercial use, distribution, and reproduction in any medium, provided the original work is properly cited. www.e-aaps.org sue, subglandular placement is undesirable. Submuscular augmentation with an implant can produce a "Snoopy's nose" deformity (double contour deformity) due to ptosis of the breast tissue over the implant [1,2]. Augmentation of the grade II ptotic breast will result in a "double-bubble" appearance if the nipple-areolar complex (NAC) is not also elevated with a skin excision [3]. Many women with ptosis undergoing augmentation may avoid a mastopexy if the nipple sits at or above the inframammary fold, the nipple and areola are visible on the front of the breast, some non-areolar skin is also visible on the front when the patient is upright, the breast overhangs the fold by no more than $2 \mathrm{~cm}$, and the nipple to inframammary fold length is no more than $8 \mathrm{~cm}$ [3].

Type III dual plane augmentation provides lower pole expansion, and results in upward rotation of the NAC without mastopexy (Fig. 1) [4]. A shaped form-stable implant may help achieve the desired breast contour by preferentially adding volume to the 

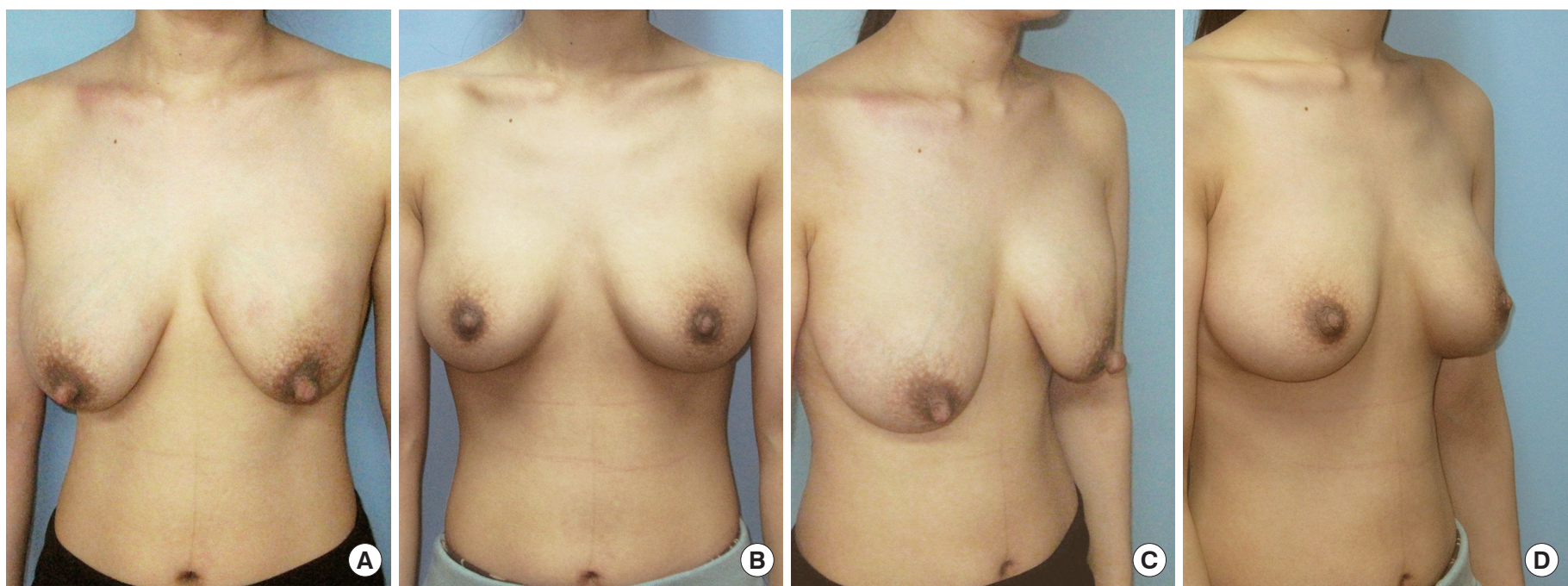

Fig. 1. This patient has operation history of simple subpectoral breast augmentation with $250 \mathrm{cc}$ smooth saline implant 6 years ago. The shape of her breasts are changed after 2 deliveries of babies. The shape of her breasts are well improved after changing pocket from simple subpectoral to type III dual plane with 250 cc smooth cohesive gel implants. (A, C) Before correction. Both breasts show Grade II ptotic appearance. Left breast shows “Snoopy appearance or Waterfall deformity". (B, D) 2 years after changing the space from simple submuscular to the type III dual plane space without masotpexy, breast implants can fill out flat envelope of lower pole of breasts and nipple areolar complex can be rotated up.
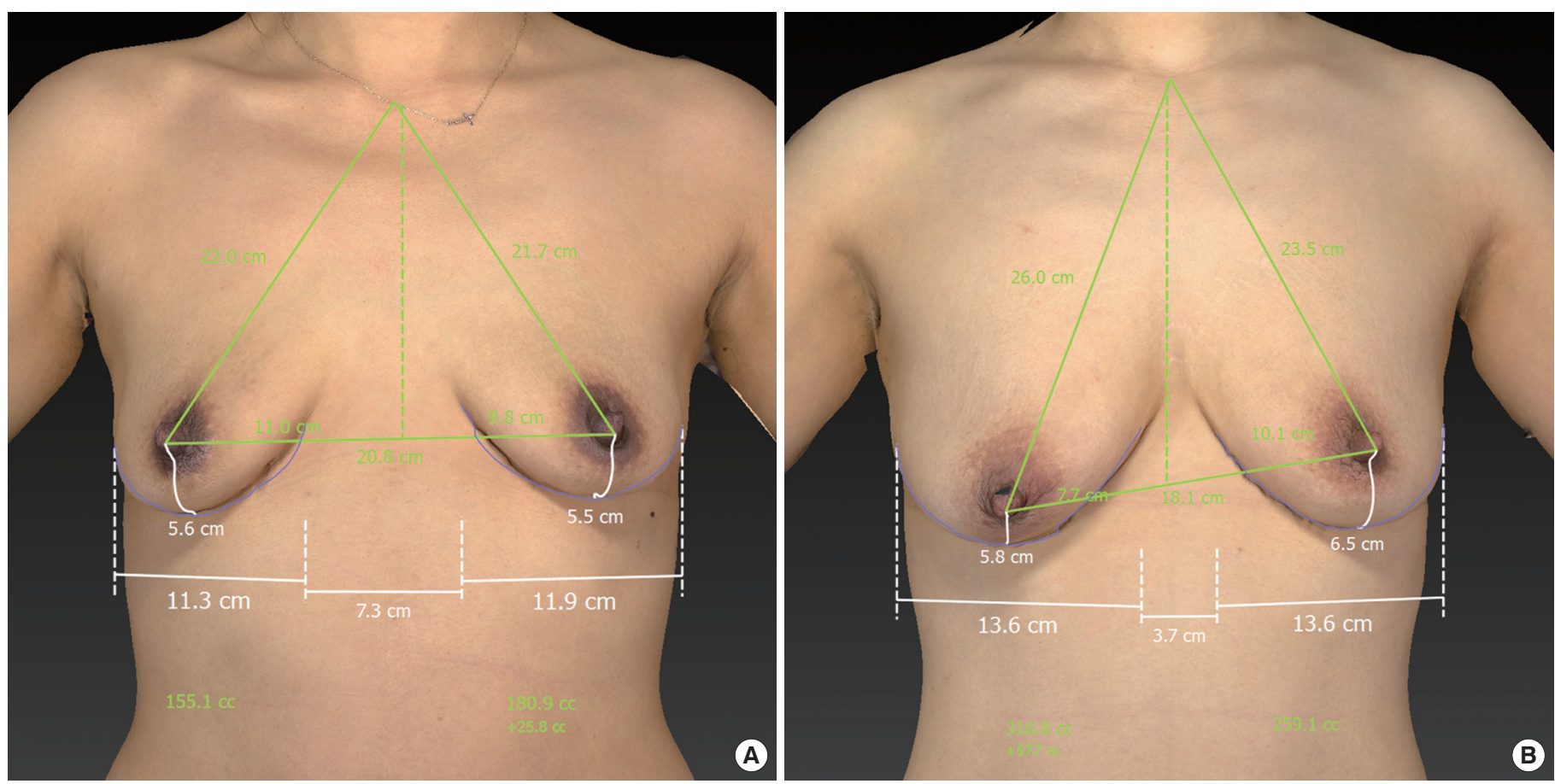

Fig. 2. Preoperative measurements of cases. (A) Case $1 . \mathrm{SSN}-\mathrm{N}$ is $22.0 \mathrm{~cm}$ and $21.7 \mathrm{~cm}$. She got relatively narrow breast width and wide intermammary distance. (B) Case $2 . \mathrm{SSN}-\mathrm{N}$ is $26 \mathrm{~cm}$ and $23.5 \mathrm{~cm}$.

inferior pole of the breast $[5,6]$. I have used shaped form-stable implants for enhancing the lower pole projection. Full height implants are used for tall, ptotic patients or those with a long distance from the suprasternal notch to nipple. Using this technique, the projection of the implant is varied according to the volume de- sired. In most cases, reasonable results are achieved with any type of anatomically-shaped implant; however, this is not always true. I herein describe 2 patients in whom improvements in rotating the NAC upward occurred by changing the implant from a full height type of implant to a moderate height type. 

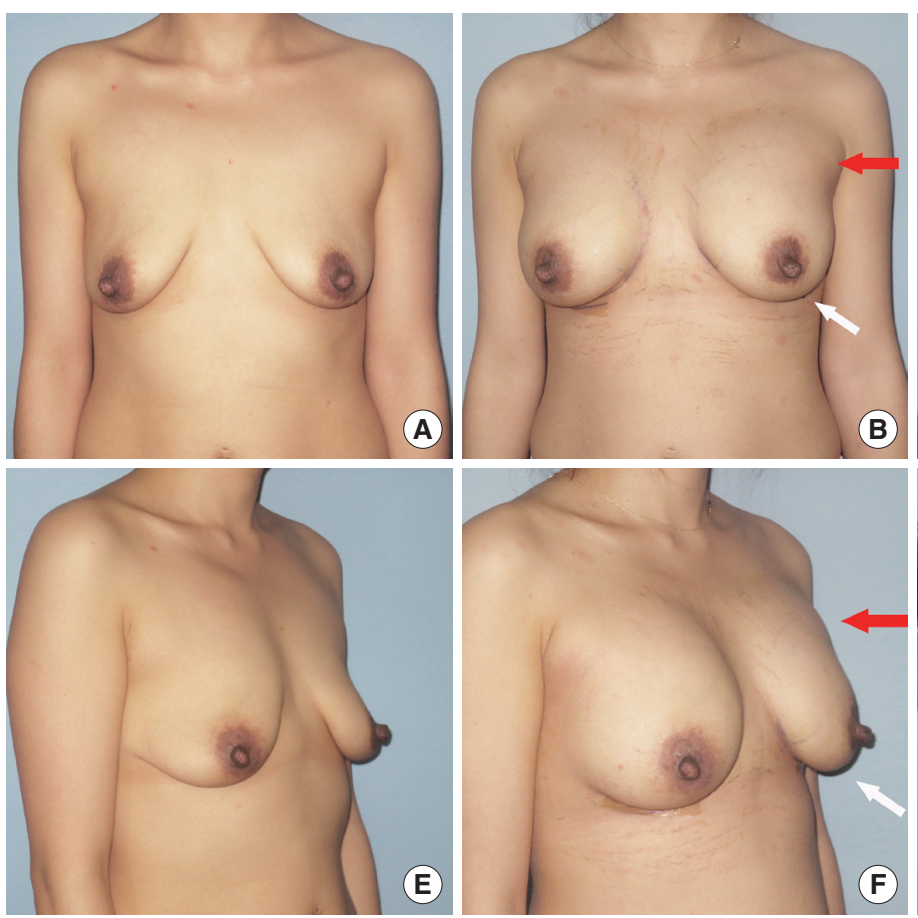
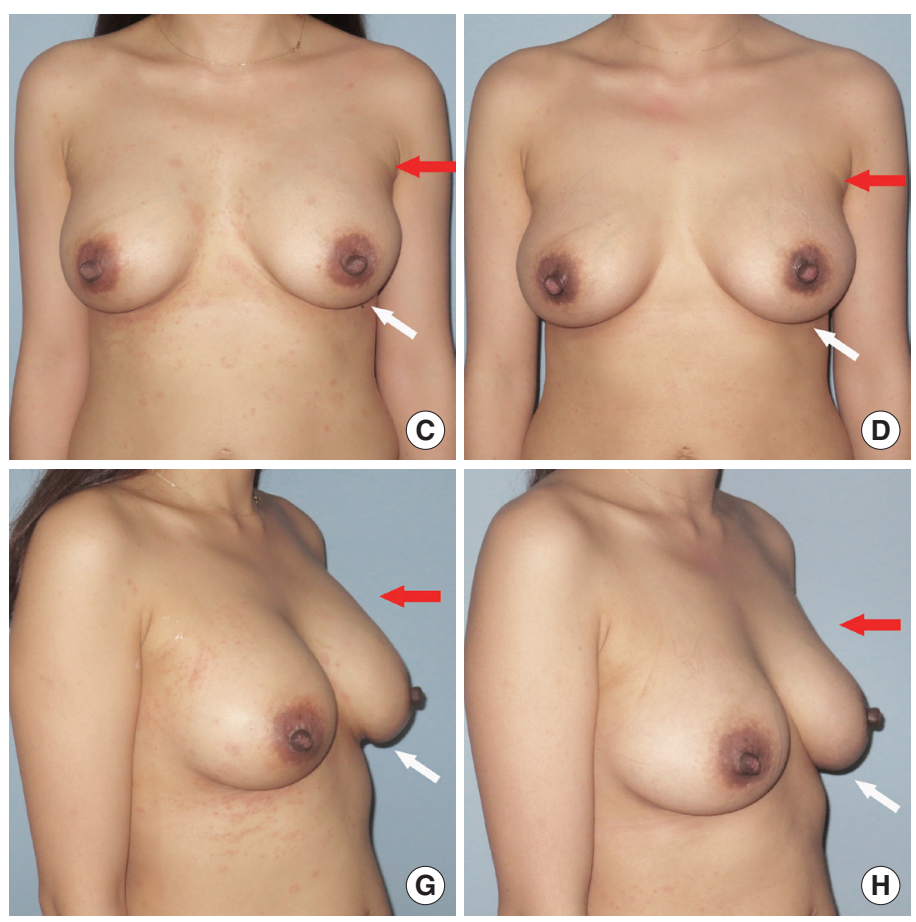

Fig. 3. Case 1. (A, E) Before operation. (B, F) POD 3 days after operation with FM350 and just before changing implants to MF335 g. Upper pole is protruded due to swelling (red arrow). Lower pole of breast is inadequately filled (white arrow). (C, G) POD 7 days after changing to MF335 g. Bulging in upper pole is diminished (red arrow). Lower pole is filled more volume (white arrow). (D, H) 3 months after changing the implant with MF335. The volume distribution is well improved (red and white arrows). Leverage effect on the lower pole is more efficient.

\section{METHODS}

Two patients initially underwent insertion of breast implants for ptotic breasts, using full height type of implants inserted using the type III dual plane technique. Both patients had wanted breasts that were not excessively large, but which had an improved shape and minimal scars. At nearly the same time after the initial surgery (postoperative day [POD] 3 and 7), each patient exhibited a unilateral double contour deformity, so I changed the implants to shift the volume to the lower pole.

\section{Case 1}

Case 1 is a 32 -year-old, $52 \mathrm{~kg}, 165 \mathrm{~cm}$ woman. Originally, the distance between her suprasternal notch and nipple (SSN-N) on the right was $22.0 \mathrm{~cm}$ and on the left was $21.7 \mathrm{~cm}$ (Fig. 2A). She had a relatively narrow breast width and a wide intermammary distance. She initially underwent breast augmentation via the type III dual plane method, with a full height moderate projection Allergan FM350 g implant (height $13 \mathrm{~cm}$, width $12.5 \mathrm{~cm}$, and projection 4.6 $\mathrm{cm})$. On POD 3, the left breast tissue envelope drooped over the breast mound. Filling and expansion between the nipple and inframammary fold were inadequate (Fig. 3B, F). The breast required more leverage effect, so I changed the implants to a moderate height full projection Allergan MF335 g implant (height $11.6 \mathrm{~cm}$, width $12.5 \mathrm{~cm}$, and projection $5.1 \mathrm{~cm}$ ).

\section{Case 2}

Case 2 is a 41 -year-old, $55 \mathrm{~kg}, 164 \mathrm{~cm}$ woman. Her original SSN-N distance on the right was $26 \mathrm{~cm}$ and on the left was $23.5 \mathrm{~cm}$ (Fig. $2 B)$. She initially underwent augmentation using the type III dual plane technique with a tall height moderate profile type Mentor 331-300 cc implant (height $12.3 \mathrm{~cm}$, width $12 \mathrm{~cm}$, and projection $3.9 \mathrm{~cm}$ ) and crescenteric mastopexy. On POD 7, filling and expansion between the nipple and inframammary fold of the right breast were inadequate. A Snoopy nose deformity was noted, and it was expected to worsen as the swelling resolved (Fig. 4A). The breast required more leverage effect, so I changed the right breast implant to a medium height moderate plus profile Mentor $322-330 \mathrm{cc} \mathrm{im}$ plant (height $11.3 \mathrm{~cm}$, width $12.0 \mathrm{~cm}$, and projection $5.1 \mathrm{~cm}$ ).

\section{RESULTS}

\section{Case 1}

On POD 7 after changing to the MF $335 \mathrm{~g}$ implant, the bulging in the upper pole of the left breast was less, and the lower pole had more volume than prior to changing the implant (Fig. 3C, G). Three months after insertion of the MF $335 \mathrm{~g}$ implant, the breast volume distribution remained improved (Fig. 3D, H).

Case 2

Three months after changing to the $322-300 \mathrm{cc}$ implant, the lower 

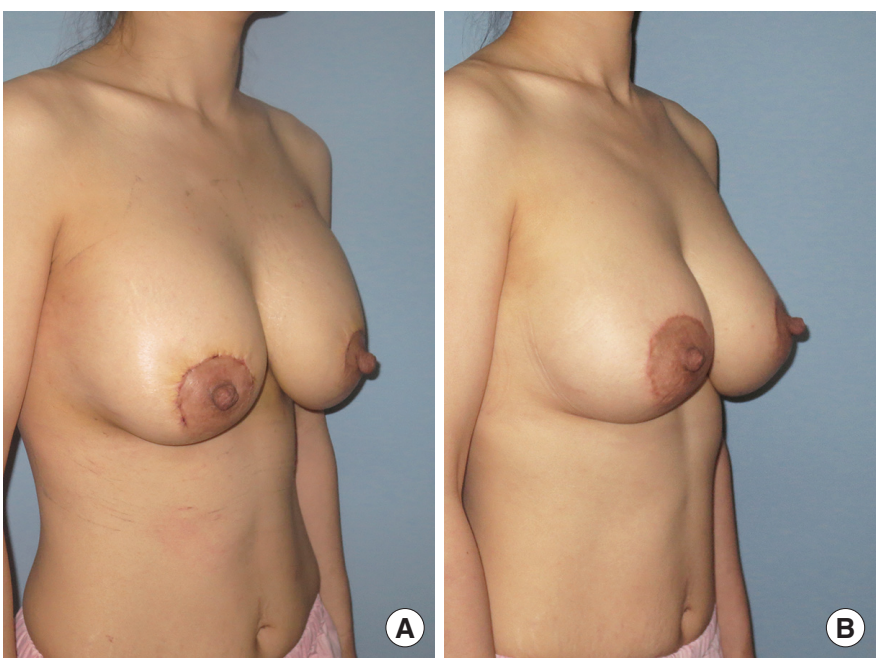

Fig. 4. Case 2. (A) Sagging the lower pole is shown 7 days after augmentation mastopexy with tall height moderate profile type Mentor 331-300 cc implant. Lower pole of breast is inadequately filled. (B) In 3 months after changing the implant to medium height moderate plus profile Mentor 322-330 cc implant, volume distribution is well improved. Leverage effect on the lower pole is more efficient.

pole of the right breast was more filled in, and the breast shape was substantially improved (Fig. 4B).

\section{DISCUSSION}

For the correction of small ptotic breasts with an implant, I follow two strategies. One strategy involves creating enough space in the lower pole of the breast to fill and expand the envelope between the nipple and the inframammary fold. For this, I choose the type III dual plane technique. The other strategy is to enhance the leverage effect of the breast to rotate the NAC upward. I choose the height of the implant according to the chest wall shape. If the patient has a long chest and lower nipple, I choose a full height type of implant. If the patient has a wide chest wall, I choose a shorter height implant. For more leverage effect, I choose the shaped implant with a shorter height and more projection.

I herein reported two patients with a double contour deformity after breast augmentation of ptotic breasts. Both initially underwent augmentation with a full height shaped implant inserted by the type III dual plane technique. The double contour deformity was successfully improved by changing the implants from a full height to moderate height style. With the same envelope and similar volume, shorter and more projectable implants have a greater leverage effect, which provides more upward rotation of the NAC (Fig. 5).

Many women are seen in consultation for ptotic breasts caused by involutional changes. Some of these can be corrected by simple augmentation, whereas others require augmentation with periare-

\section{Envelope $=A+B=a^{\prime}+a^{\prime \prime}+b$}
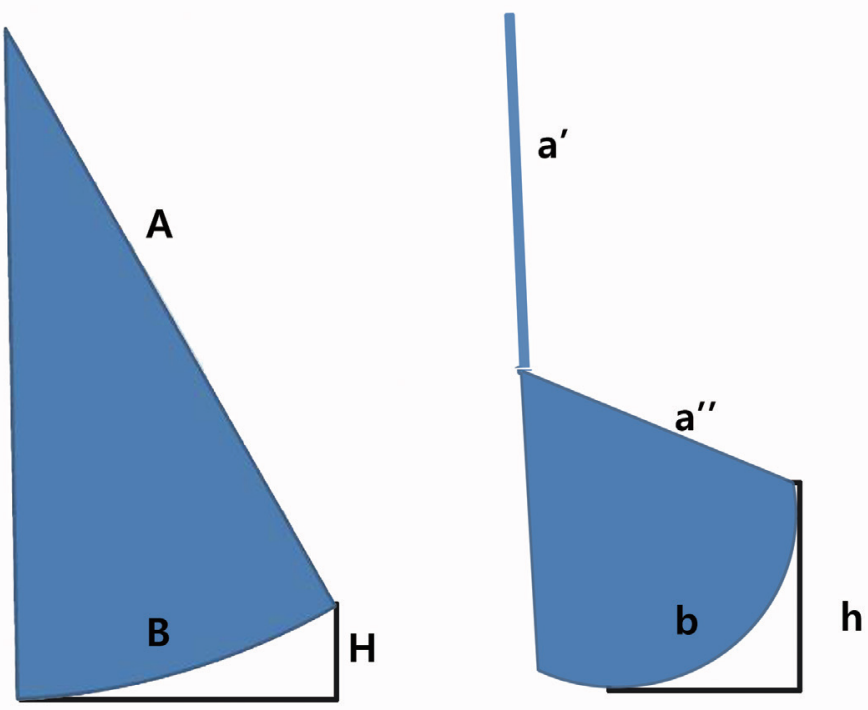

Fig. 5. Schematic comparison of leverage action between tall vs short implant with similar volume.

olar or vertical mastopexy. Many Korean women with ptosis who undergo augmentation prefer to avoid a mastopexy because they do not want a scar. Spear et al. suggested that it may be possible to perform augmentation without mastopexy if the nipple sits at or above the inframammary fold, the nipple and areola are visible on the front of the breast, some non-areola skin is also visible on the front when the patient is upright, the breast overhangs the fold by no more than $2 \mathrm{~cm}$, and the nipple to inframammary fold length is no more than $8 \mathrm{~cm}$ [3].

Submuscular augmentation of the ptotic breast can result in a "double-bubble" appearance because a submuscular implant will lay superior to the ptotic gland. Subglandular augmentation in a ptotic breast avoids the "double-bubble" situation but may aggravate the preexisting ptosis. For reducing double contour deformity, a type III dual plane technique is recommended, which involves complete division of the origins of the pectoralis muscle across the inframammary fold and dissection in the retromammary plane to the superior border of the areola [4]. The dissection in the retroglandular plane should be performed cautiously and incrementally to avoid excessive upward retraction of the pectoral muscle. The dual plane technique facilitates unfurling and redistribution of the tight skin of the inferior pole to accommodate the implant, increasing the arc length from the nipple to the inframammary fold. A shaped form-stable implant may help achieve the desired contour by preferentially adding volume to the inferior pole of the breast $[5,6]$.

\section{CONCLUSION}

For rotating the NAC upward during correction of small ptotic 
breasts with an implant, the type III dual plane technique effectively produces a greater volume pocket in the lower pole of the breast. A greater leverage effect can be obtained by using a shorter height, greater projection type of shaped implant instead of a full height implant.

\section{REFERENCES}

1. Calobrace MB, Herdt DR, Cothron KJ. Simultaneous augmentation/ mastopexy: A retrospective 5-year review of 332 consecutive cases. Plast Reconstr Surg 2013;131:145-56.

2. Kirwan L. Augmentation of the ptotic breast: Simultaneous periareo- lar mastopexy/breast augmentation. Aesthetic Surg J 1999;19:34-9.

3. Spear SL, Boehmler JH, Clemens MW. Augmentation/mastopexy: A 3-year review of a single surgeon's practice. Plast Reconstr Surg 2006; 118(Suppl):136S-47S.

4. Tebbetts JB. Dual plane breast augmentation: Optimizing implant-softtissue relationships in a wide range of breast types. Plast Reconstr Surg 2001;107:1255-72.

5. Spear SL, Bulan EJ, Venturi ML. Breast augmentation. Plast Reconstr Surg. 2006;118(Suppl):188S-96S.

6. Serra-Renom JM, Munoz-Olmo J, Serra-Mestre JM. Endoscopically assisted aesthetic augmentation of tuberous breasts and fat grafting to correct the double bubble. Aesthetic Plast Surg 2012;36:1114-9. 